Full length article

\title{
Antiparasitic evaluation of betulinic acid derivatives reveals effective and selective anti-Trypanosoma cruzi inhibitors
}

\author{
Cássio Santana Meira a, José Maria Barbosa-Filho ${ }^{\mathrm{b}}$, Adriana Lanfredi-Rangel ${ }^{\mathrm{a}}$, \\ Elisalva Teixeira Guimarães ${ }^{\text {a, c }}$, Diogo Rodrigo Magalhães Moreira ${ }^{a}$, \\ Milena Botelho Pereira Soares a, d, * \\ ${ }^{a}$ Centro de Pesquisas Gonçalo Moniz, Fundação Oswaldo Cruz (FIOCRUZ), Salvador, BA, Brazil \\ ${ }^{\mathrm{b}}$ Laboratório de Tecnologia Farmacêutica, Universidade Federal da Paraíba, João Pessoa, PB, Brazil \\ c Universidade do Estado da Bahia, Departamento de Ciências da Vida, Salvador, BA, Brazil \\ ${ }^{\mathrm{d}}$ Hospital São Rafael, Centro de Biotecnologia e Terapia Celular, Salvador, BA, Brazil
}

\section{H I G H L I G H T S}

- Terpenoids are potent and selective trypanocidal agents.

- BA5 destroys parasite cells by necrotic death.

- Betulinic acid derivatives inhibit the growth of intracellular amastigotes of T. cruzi.

- Combination of BA5 and benznidazole showed synergistic effects.

\section{A R T I C L E I N F O}

\section{Article history:}

Received 26 November 2015

Received in revised form

6 April 2016

Accepted 10 April 2016

Available online 11 April 2016

\section{Keywords:}

Chagas disease

Trypanosoma cruzi

Natural compounds

Semi-synthetic

Triterpenoids

Betulinic acid
G R A P H I C A L A B S T R A C T

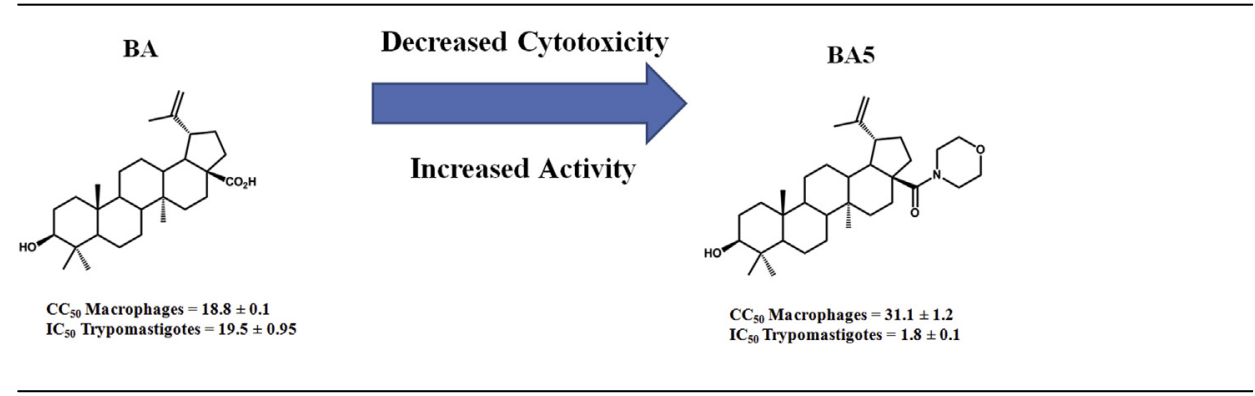

\begin{abstract}
A B S T R A C T
Betulinic acid is a pentacyclic triterpenoid with several biological properties already described, including antiparasitic activity. Here, the anti-Trypanosoma cruzi activity of betulinic acid and its semi-synthetic amide derivatives (BA1-BA8) was investigated. The anti-Trypanosoma cruzi activity and selectivity were enhanced in semi-synthetic derivatives, specially on derivatives BA5, BA6 and BA8. To understand the mechanism of action underlying betulinic acid anti-T. cruzi activity, we investigated ultrastructural changes by electron microscopy. Ultrastructural studies showed that trypomastigotes incubated with BA5 had membrane blebling, flagella retraction, atypical cytoplasmic vacuoles and Golgi cisternae dilatation. Flow cytometry analysis showed that parasite death is mainly caused by necrosis. Treatment with derivatives BA5, BA6 or BA8 reduced the invasion process, as well as intracellular parasite development in host cells, with a potency and selectivity similar to that observed in benznidazole-treated cells. More importantly, the combination of BA5 and benznidazole revealed synergistic effects on trypomastigote and amastigote forms of $T$. cruzi. In conclusion, we demonstrated that BA5 compound is an effective and selective anti-T. cruzi agent.
\end{abstract}

() 2016 Elsevier Inc. All rights reserved.

\footnotetext{
* Corresponding author. Centro de Pesquisas Gonçalo Moniz, Fundação Oswaldo Cruz, Rua Waldemar Falcão, 121, Candeal, Salvador 40296-710, BA, Brazil.

E-mail address: milena@bahia.fiocruz.br (M.B.P. Soares).
}

\section{Introduction}

Chagas disease is a neglected disease caused by the protozoan parasite Trypanosoma cruzi and constitutes a serious public health problem worldwide (Pinto-Dias, 2006). It affects about 8-10 
million people, mainly in Latin American countries, where this disease is endemic (Rassi et al., 2010). Pharmacotherapy is based on nifurtimox and benznidazole, which are recommended to treat all infected people (Urbina and Docampo, 2003). Treatment with benznidazole is associated with side effects, prolonged treatment time and low and variable efficacy in chronic phase of infection, which is the most prevalent form of the disease (Urbina and Docampo, 2003; Viotti et al., 2009; Morillo et al., 2015). This scenario emphasizes a need to develop safer and more effective drugs.

Natural products play an important role in drug discovery and development (Newman and Cragg, 2012). Naturally occurring terpenoids represent an important class of bioactive compounds that exhibit several medicinal properties (Yin, 2015). This is exemplified by betulinic acid, a lupane-type pentacyclic triterpenoid abundant in the plant kingdom, which can be isolated from several plant species or obtained from its metabolic precursor, betulin (Yogeeswari and Sriram, 2005). Betulinic acid and its derivatives possess anti-HIV activity, anti-bacterial, antihelmitic, antiinflammatory and a potent cytotoxic activity against a large panel of tumor cell lines (Baglin et al., 2003; Chandramu et al., 2003; Yogeeswari and Sriram, 2005; Drag et al., 2009; Costa et al., 2014; Chakraborty et al., 2015).

Betulinic acid and other triterpenoids, both naturally-occurring and semi-synthetic, have also been investigated as antiparasitic agents (Hoet et al., 2007; Innocente et al., 2012). More specifically, the activity of betulinic acid and its derivatives against the erythrocitic stage of a chloroquine-resistant Plasmodium falciparum strain, as well as antileishmanial activity on different Leishmania species, were reported (Alakurtti et al., 2010; Chen et al., 2010; Innocente et al., 2012; Sousa et al., 2014). Regarding the anti-Trypanosoma cruzi activity, it was previously shown that betulinic acid and ester derivatives inhibit epimastigote proliferation (Domínguez-Carmona et al., 2010). In view of these findings, betulinic acid is considered to be a prototype for the design and synthesis of antiprotozoal agents. Chemical modifications of the carboxyl group have suggested that this part of the molecule can produce derivatives with enhanced antiprotozoal activity when compared to betulinic acid (Gros et al., 2006; Domínguez-Carmona et al., 2010; Da Silva et al., 2011; Sousa et al., 2014; Spivak et al., 2014). Based on these facts, the purpose of our work was to evaluate the trypanocidal potential of new semi-synthetic amide derivatives of betulinic acid.

\section{Material and methods}

\subsection{Chemistry}

Betulinic acid (BA) was extracted from the bark of Ziziphus joazeiro Mart. (Rhamnaceae) by using a previously described method (Barbosa-Filho et al., 1985). Semi-synthetic compounds (BA1 to BA8) were prepared from betulinic acid (Fig. 1). Betulinic acid was initially converted to mixed anhydride by using isobutyl chloroformate (Sigma-Aldrich), followed by addition of the respective secondary amines. This methodology allowed the synthesis of compounds BA1 to BA8 (Fig. 1), with yields varying from 30 to $41 \%$ after HPLC purification.

\subsection{General procedure for the synthesis}

Two mmol $(0.1 \mathrm{~g})$ of betulinic acid were added to a $100 \mathrm{~mL}$ round bottom flask under magnetic stirring and dissolved in dichloromethane $(10 \mathrm{~mL})$. The mixture was cooled to $-10{ }^{\circ} \mathrm{C}$ and then 4-dimethylaminopiridine isobutyl chloroformate $(3.0 \mathrm{mmol}$, $0.4 \mathrm{~g})$ in dichloromethane $(1 \mathrm{~mL})$ was slowly added during $30 \mathrm{~min}$. The mixture was maintained under stirring for $3 \mathrm{~h}$. The reaction was transferred to a separating funnel, to which $50 \mathrm{~mL}$ of ethyl ether were added and the organic phase was quickly washed with a saturated solution of sodium bisulfite $(1 \times 50 \mathrm{~mL})$, washed with water and with saturated $\mathrm{NaCl}$ solution $(2 \times 50 \mathrm{~mL})$. The organic phase was separated, dried over $\mathrm{MgSO}_{4}$, and evaporated under reduced pressure to give a white thick oily residue, which was crystallized in cyclohexane. Purity was analyzed by HPLC and structures examined by HRESIMS. Mass spectrometry was performed in a Q-TOF spectrometer (nanoUPLC-Xevo G2 Tof, Waters). ESI was carried out in the positive ion mode. HPLC analysis was carried out in Beckmann Coulter using UV detector in a $\mathrm{C}_{18}$ column $(100 \AA$, $2.14 \times 25 \mathrm{~cm})$ with a linear gradient of $5-95 \% \mathrm{MeCN} / \mathrm{H}_{2} \mathrm{O}$ in 0.1\% TFA. Compound BA1. 95\% (HPLC). HRESIMS Anal. Calc. (Found)/ Error for $\mathrm{C}_{34} \mathrm{H}_{55} \mathrm{NO}_{2}$ : $509.4232\left(510.4713,\left[\mathrm{M}+\mathrm{H}^{+}\right]\right) / 6.5 \mathrm{ppm}$. Compound BA2. 94\% (HPLC). HRESIMS Anal. Calc. (Found)/Error for $\mathrm{C}_{35} \mathrm{H}_{58} \mathrm{~N}_{2} \mathrm{O}_{2}$ : 538.4498 (539.4491, $\left.\left[\mathrm{M}+\mathrm{H}^{+}\right]\right) / 5.7 \mathrm{ppm}$. Compound BA3. 98\% (HPLC). HRESIMS Anal. Calc. (Found)/Error for $\mathrm{C}_{35} \mathrm{H}_{57} \mathrm{NO}_{2}$ : 523.4389 (524.5031, $\left.\left[\mathrm{M}+\mathrm{H}^{+}\right]\right) / 5.0$ ppm. Compound BA4. 98\% (HPLC). HRESIMS Anal. Calc. (Found)/Error for $\mathrm{C}_{34} \mathrm{H}_{56} \mathrm{~N}_{2} \mathrm{O}_{2}$ : 524.4341 (524.4249, $\left.\left[\mathrm{M}+\mathrm{H}^{+}\right]\right) / 4.0$ ppm. Compound BA5. 95\% (HPLC). HRESIMS Anal. Calc. (Found)/Error for $\mathrm{C}_{33} \mathrm{H}_{53} \mathrm{NO}_{3}: 511.4025$ (512.3177, $\left.\left[\mathrm{M}+\mathrm{H}^{+}\right]\right) / 10$ ppm. Compound BA6. 94\% (HPLC). HRESIMS Anal. Calc. (Found)/Error for $\mathrm{C}_{33} \mathrm{H}_{53} \mathrm{NSO}_{2}$ : 527.3797 (529.1641, $\left.\left[\mathrm{M}+\mathrm{H}^{+}\right]\right) / 20$ ppm. Compound BA7. 95\% (HPLC). HRESIMS Anal. Calc. (Found)/Error for $\mathrm{C}_{40} \mathrm{H}_{58} \mathrm{NFO}_{2}$ : 603.4451 (604.4085, $\left[\mathrm{M}+\mathrm{H}^{+}\right]$)/ 4.0 ppm. Compound BA8. 98\% (HPLC). HRESIMS Anal. Calc. (Found)/ Error for $\mathrm{C}_{40} \mathrm{H}_{59} \mathrm{NO}_{2}$ : $585.4545\left(589.7109,\left[\mathrm{M}+\mathrm{H}^{+}\right]\right) / 10 \mathrm{ppm}$.

\subsection{Cytotoxicity to mammalian cells}

Peritoneal exudate macrophages were obtained by washing, with cold RPMI medium, the peritoneal cavity of BALB/c mice 4-5 days after injection of $3 \%$ thioglycolate in saline ( $1.5 \mathrm{~mL}$ per mice). Then, cells were placed into 96-well plates at a density $1 \times 10^{5}$ cells/well in RPMI-1640 medium without phenol red (Sigma-Aldrich, St. Louis, MO) supplemented with $10 \%$ of fetal bovine serum (FBS; Cultilab, Campinas, Brazil), and $50 \mu \mathrm{g} / \mathrm{mL}$ of gentamycin (Novafarma, Anápolis, Brazil) and incubated for $24 \mathrm{~h}$ at $37{ }^{\circ} \mathrm{C}$ and $5 \% \mathrm{CO}_{2}$. After that time, each compound was added in triplicate at eight concentrations ranging from 0.04 to $100 \mu \mathrm{M}$ and incubated for 6 or $72 \mathrm{~h}$. Twenty $\mu \mathrm{L} /$ well of AlamarBlue (Invitrogen, Carlsbad, CA) was added to the plates during $10 \mathrm{~h}$. Colorimetric readings were performed at 570 and $600 \mathrm{~nm}$. $\mathrm{CC}_{50}$ values were calculated using data-points gathered from three independent experiments. Gentian violet (Synth, São Paulo, Brazil) was used as a cytotoxicity control, at concentrations ranging from 0.04 to $10 \mu \mathrm{M}$.

\subsection{Cytotoxicity for trypomastigotes}

Bloodstream trypomastigotes forms of $T$. cruzi (Y strain) were obtained from supernatants of LLC-MK2 cells previously infected and maintained in RPMI-1640 medium supplemented with $10 \%$ FBS, and $50 \mu \mathrm{g} / \mathrm{mL}$ gentamycin at $37{ }^{\circ} \mathrm{C}$ and $5 \% \mathrm{CO}_{2}$. Parasites $\left(4 \times 10^{5}\right.$ cells/well) were dispensed into 96 -well plates and the test inhibitors were added at eight concentrations ranging from 0.04 to $100 \mu \mathrm{M}$ in triplicate, and the plate was incubated for $24 \mathrm{~h}$ at $37{ }^{\circ} \mathrm{C}$ and $5 \%$ of $\mathrm{CO}_{2}$. Aliquots of each well were collected and the number of viable parasites was assessed in a Neubauer chamber and compared to untreated cultures. Benznidazole (LAFEPE, Recife, Brazil) was used as positive control in the anti-Trypanosoma cruzi studies. Three independent experiments were performed.

\subsection{In vitro $T$. cruzi infection assay}

Peritoneal exudate macrophages were plated at a cell density of 


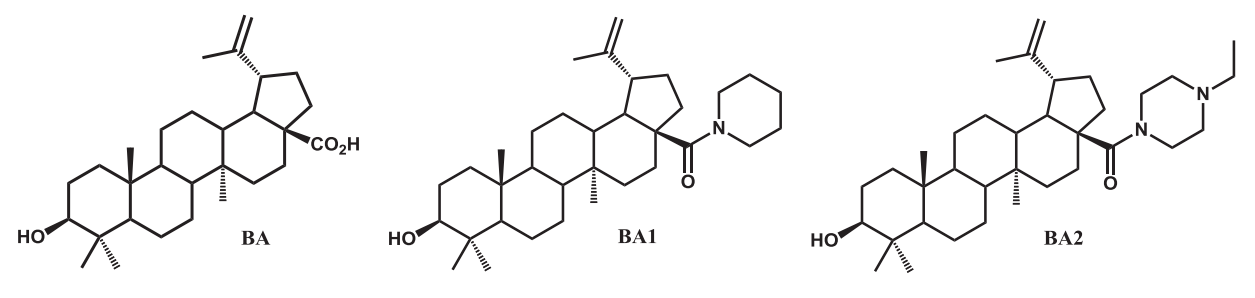

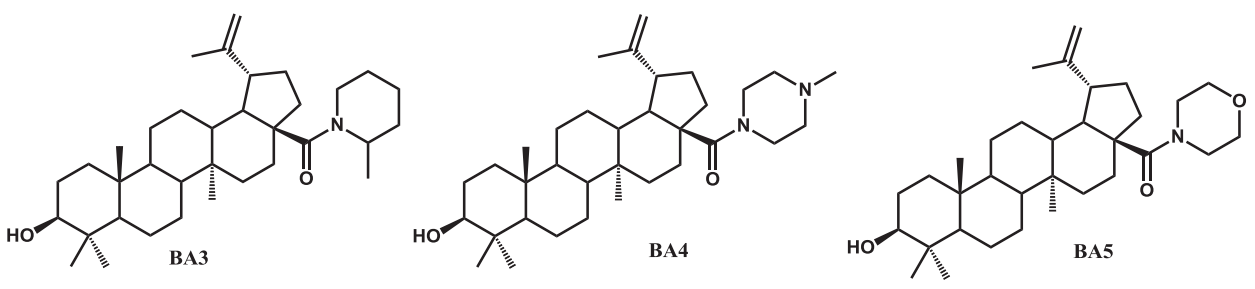<smiles>C=C(C)C1CCC2(C(=O)N3CCSCC3)CCC1C1CCC3C(CCC4C(C)(C)C(O)CCC34C)[C@]1(C)CC2</smiles>

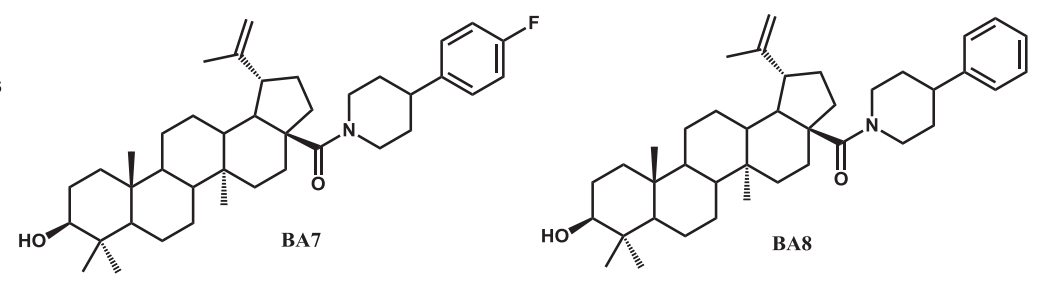

Fig. 1. Structure of betulinic acid and its derivatives.

$2 \times 10^{5}$ cells/well in 24-well plates with sterile coverslips on the bottom in RPMI supplemented with 10\% FBS and incubated for $24 \mathrm{~h}$ at $37{ }^{\circ} \mathrm{C}$ and $5 \% \mathrm{CO}_{2}$. Cells were then infected with trypomastigotes at a ratio of 10 parasites per macrophage for $2 \mathrm{~h}$. Free trypomastigotes were removed by successive washes using saline solution. Cultures were incubated in complete medium alone or with the compounds under investigation in different concentrations for $6 \mathrm{~h}$. The medium was replaced with fresh medium and the plate was incubated for 3 days (Soares et al., 2012). Cells were fixed in absolute alcohol and the percentage of infected macrophages and the mean number of amastigotes/100 macrophages was determined by manual counting after hematoxylin and eosin staining in an optical microscope (Olympus, Tokyo, Japan). The percentage of infected macrophages and the relative number of amastigotes per macrophage was determined by counting 100 cells per slide. Experiments were performed three times.

\subsection{Trypanosoma cruzi invasion assay}

Peritoneal exudate macrophages $\left(10^{5}\right.$ cells) were plated onto sterile coverslips in 24-well plates and kept for $24 \mathrm{~h}$. Plates were washed with saline solution and trypomastigotes were then added at a cell density of $1 \times 10^{7}$ parasites/well along with the addition of BA5, or BA6 or BA8 $(50 \mu \mathrm{M})$. The plate was incubated for $2 \mathrm{~h}$ at $37^{\circ} \mathrm{C}$ and $5 \% \mathrm{CO}_{2}$, followed by successive washes with saline solution to remove extracellular trypomastigotes. Plates were maintained in RPMI medium supplemented with $10 \% \mathrm{FBS}$ at $37^{\circ} \mathrm{C}$ for $2 \mathrm{~h}$. Infected macrophages were examined for the presence of amastigotes by optical microscopy using a standard hematoxylin and eosin staining. Amphotericin B (Gibco Laboratories, Gaithersburg, MD) was used as a positive control in this assay. Three independent experiments were performed.

\subsection{Ultrastructural studies}

Trypomastigotes at a cell density of $1 \times 10^{7}$ cells/mL in 24 wellplates were treated with test inhibitor BA5 $(2$ or $4 \mu \mathrm{M})$ or not for
$24 \mathrm{~h}$. Parasites were then fixed with $2 \%$ formaldehyde and 2.5\% glutaraldehyde (Electron Microscopy Sciences, Hatfield, PA) in sodium cacodylate buffer (0.1 M, pH 7.2) for $1 \mathrm{~h}$ at room temperature. After fixation, parasites were washed 3 times with sodium cacodylate buffer ( $0.1 \mathrm{M}, \mathrm{pH} 7.2)$, and post-fixed with a $1.0 \%$ solution of osmium tetroxide containing $0.8 \%$ potassium ferrocyanide (Sigma) for $1 \mathrm{~h}$. Cells were subsequently dehydrated in increasing concentrations of acetone (30, 50, 70, 90 and 100\%) for 10 min at each step and embedded in polybed resin (PolyScience family, Warrington, PA). Ultrathin sections on copper grids were contrasted with uranyl acetate and lead citrate and observed under a ZEISS 109 transmission electron microscope. For scanning electron microscopy, trypomastigotes treated with or without BA $505(2$ or $4 \mu \mathrm{M})$ and fixed in the same conditions were washed in $0.1 \mathrm{M}$ cacodylate buffer, and allowed to adhere in coverslips pre-coated with poly-Llysine (Sigma). Cells were then post-fixed with a solution of osmium tetroxide containing $0.8 \%$ of potassium ferrocyanide for $30 \mathrm{~min}$ and dehydrated in crescent concentrations of ethanol (30, 50, 70, 90 and 100\%). The samples were dried until the critical point, metallized with gold and analyzed in a JEOL JSM-6390LV scanning electron microscope. Two independent experiments were performed.

\subsection{Propidium iodide and annexin $V$ staining}

Trypomastigotes $1 \times 10^{7} / \mathrm{mL}$ in 24 well-plates were treated with 5 or $10 \mu \mathrm{M}$ of BA5 in RPMI supplemented with FBS at $37^{\circ} \mathrm{C}$ for 24 or $72 \mathrm{~h}$ and labeled for propidium iodide (PI) and annexin $\mathrm{V}$ using the annexin V-FITC apoptosis detection kit (Sigma), according to the manufacturer's instructions. Acquisition and analyses was performed using a FACS Calibur flow cytometer (Becton Dickinson, San Diego, CA), with FlowJo software (Tree Star, Ashland, OR). A total of 10,000 events were acquired in the region previously established as that corresponding to trypomastigotes forms of T. cruzi. Two independent experiments were performed. 
Table 1

Cytotoxicity against macrophages and anti-Trypanosoma cruzi activity against trypomastigotes forms of betulinic acid and its derivatives.

\begin{tabular}{lllc}
\hline Compound & $\mathrm{CC}_{50}(\mu \mathrm{M})^{\mathrm{a}}$ & $\mathrm{IC}_{50}$ Try. $(\mu \mathrm{M})$ & SI \\
\hline BA & $18.8( \pm 0.1)$ & $19.5( \pm 0.9)$ & 0 \\
BA1 & $>100$ & $>100$ & - \\
BA2 & $>100$ & $>100$ & - \\
BA3 & $>100$ & $13.7( \pm 2.3)$ & 7.3 \\
BA4 & $39.7( \pm 0.5)$ & $10.2( \pm 1.0)$ & 3.9 \\
BA5 & $31.1( \pm 1.2)$ & $1.8( \pm 0.1)$ & 17.3 \\
BA6 & $28.7( \pm 1.1)$ & $5.4( \pm 1.3)$ & 5.3 \\
BA7 & $>100$ & $55( \pm 0.6)$ & 1.8 \\
BA8 & $53.5( \pm 0.4)$ & $5( \pm 0.5)$ & 10.7 \\
Gentian violet & $0.5( \pm 0.1)$ & - & - \\
Benznidazole & $>100$ & $11.4( \pm 1.4)$ & 8.8 \\
\hline
\end{tabular}

$\mathrm{CC}_{50}=$ cytotoxicity concentration $50 \% . \mathrm{IC}_{50}=$ inhibitory concentration. $\mathrm{SI}=$ Selective index. $50 \%$. Values are means \pm SD of three independent experiments performed in triplicate.

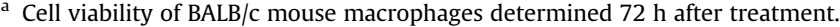

\subsection{Drug combination}

For in vitro drug combinations, doubling dilutions of each drug (BA5 and benznidazole), used alone or in fixed combinations were incubated with trypomastigotes or intracellular parasites followed the protocols described above. The analysis of the combined effects was performed by determining the combination index $(\mathrm{CI})$, used as cutoff to determine synergism, by using Chou-Talalay CI method (Chou and Talalay, 2005) and through the construction of isobologram using the fixed ratio method, as described previously (Fivelman et al., 2004).

\subsection{Statistical analyses}

To determine the cytotoxicity concentration $50 \%$ of BALB/c mice macrophages $\left(\mathrm{CC}_{50}\right)$ and the inhibitory concentration $50 \%\left(\mathrm{IC}_{50}\right)$ of the trypomastigotes and amastigotes forms of $T$. cruzi, we used nonlinear regression. The selectivity index (SI) was defined as the ratio of $\mathrm{CC}_{50}$ by $\mathrm{IC}_{50}$ (trypomastigotes or amastigotes). The one-way ANOVA followed by Bonferroni's multiple comparison test was used to determine the statistical significance of the group comparisons in the in vitro infection studies and cell invasion study. Results were considered statistically significant when $P<0.05$. All analyses were performed using Graph Pad Prism version 5.01 (Graph Pad Software, San Diego, CA).

\section{Results}

\subsection{Trypanocidal and cytotoxicity activity}

First the compounds had their anti-T. cruzi activity determined against trypomastigote forms (Table 1 ). The structural design of the
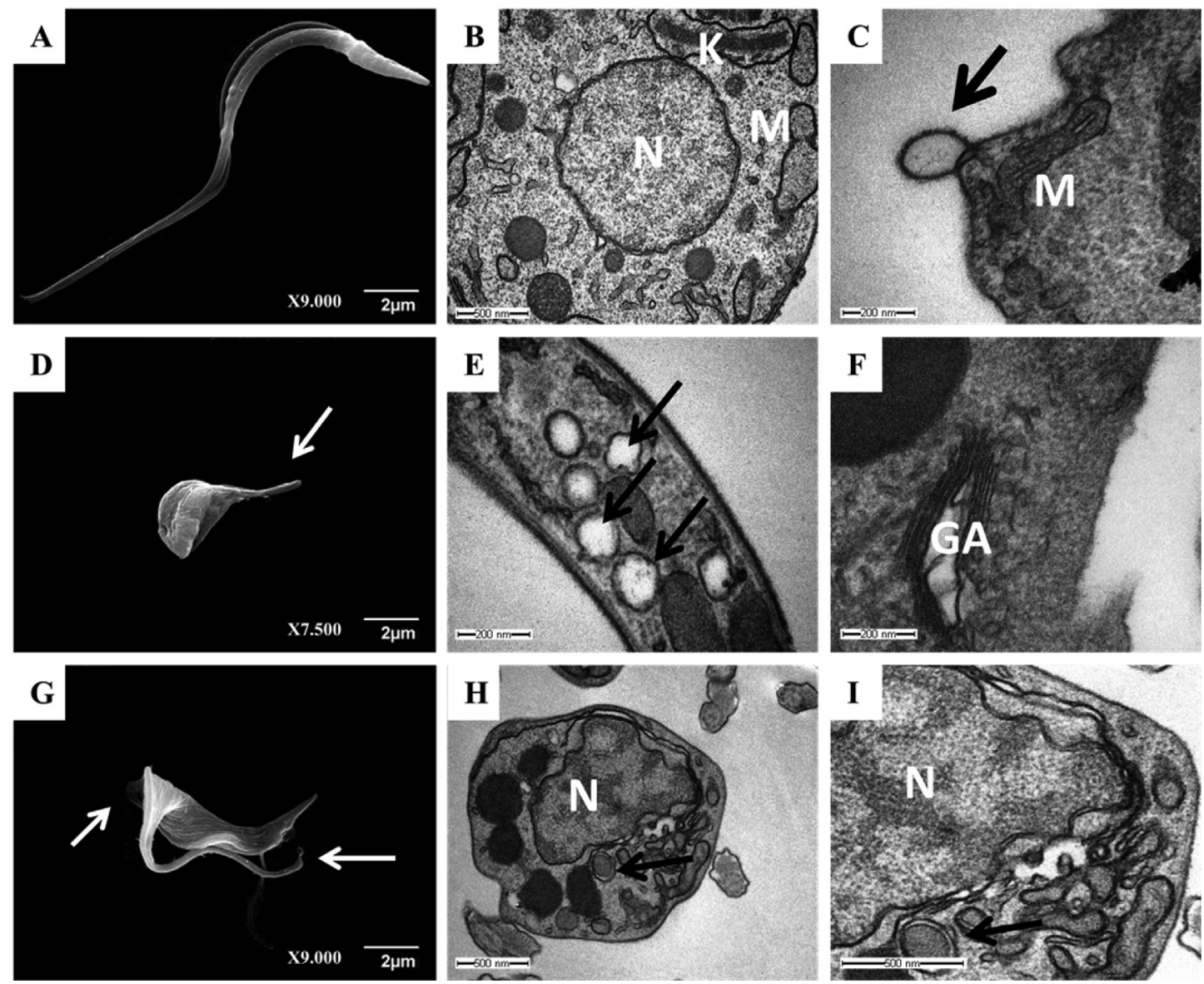

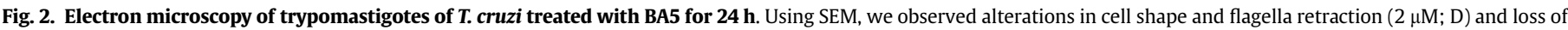

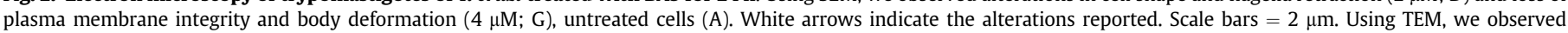

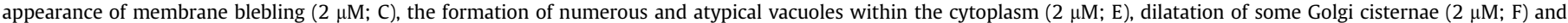

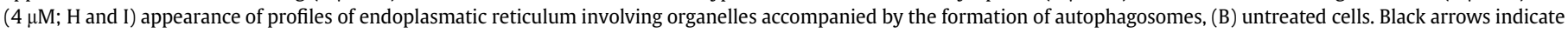
changes in the organelles. 

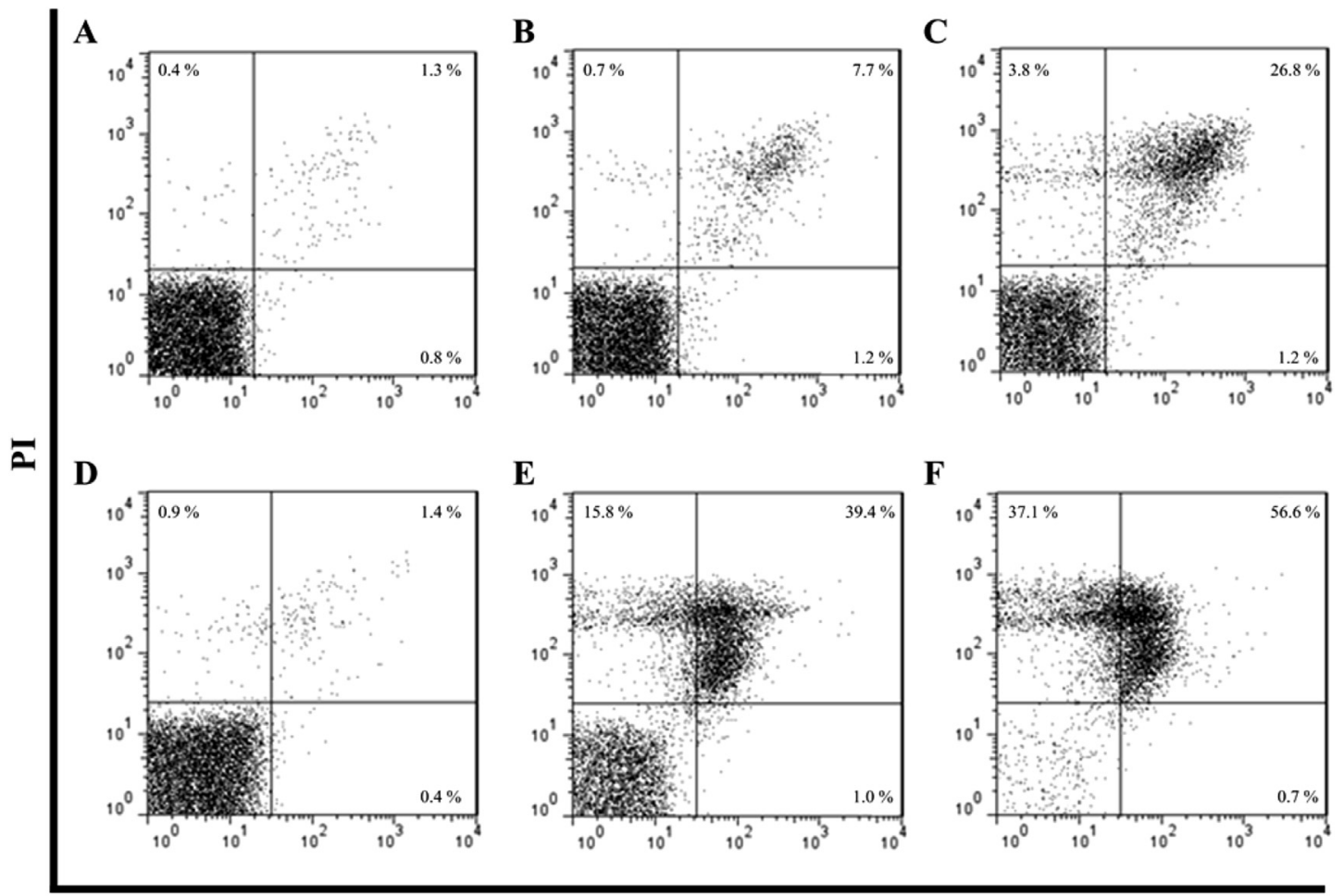

Annexin V

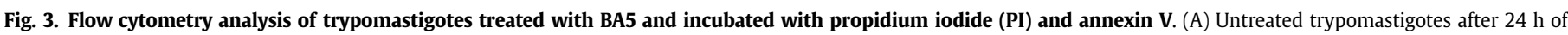

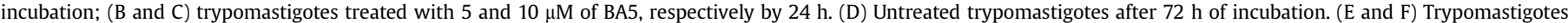
treated with 5 or $10 \mu \mathrm{M}$ of BA5, respectively, for $72 \mathrm{~h}$.
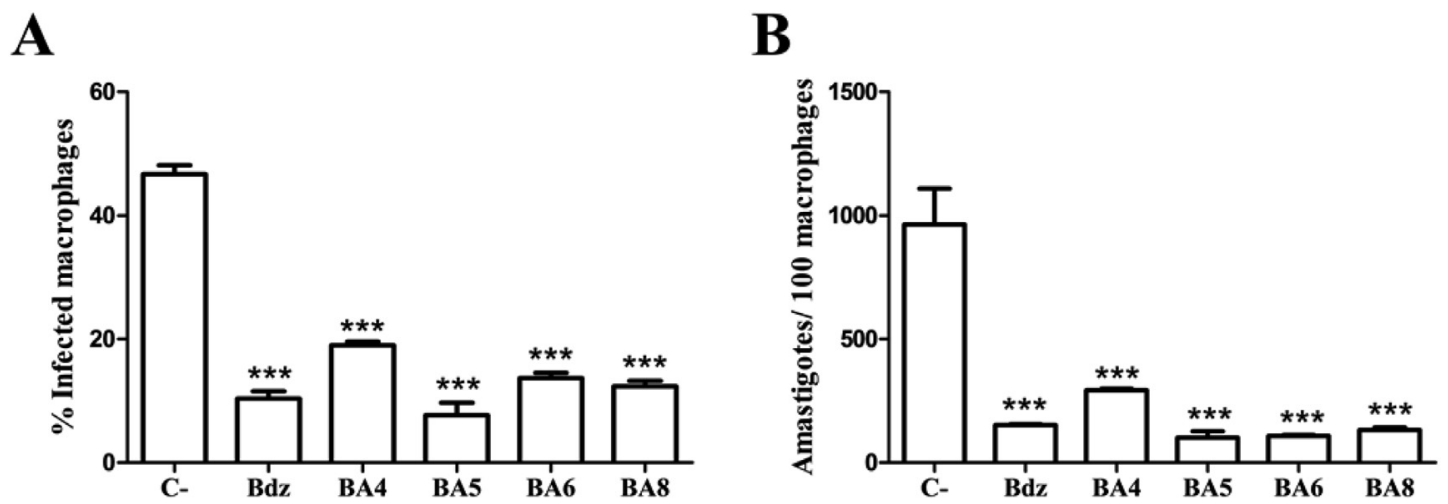

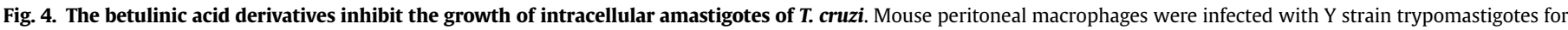

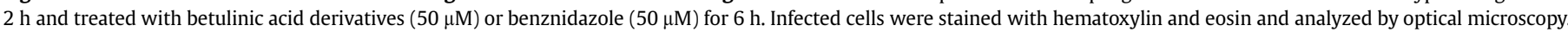
$\mathrm{Bdz}=$ benznidazole. Values represent the mean \pm SEM of triplicate. ${ }^{* * *}, P<0.001$ compared with untreated cultures.

Table 2

Antiparasitic activity in intracellular parasite, host cells cytotoxicity and selectivity index of derivatives.

\begin{tabular}{llll}
\hline Comp. & Amastigotes $\mathrm{IC}_{50} \pm$ S.D. $(\mu \mathrm{M})^{\mathrm{a}}$ & ${\text { Macrophages } \mathrm{CC}_{50} \pm \text { S.D. }(\mu \mathrm{M})^{\mathrm{b}}}$ & Selectivity index $^{\mathrm{c}}$ \\
\hline BA4 & $18.9( \pm 1.2)$ & $>100$ & $>5.3$ \\
BA5 & $10.6( \pm 0.8)$ & $>100$ & $>9.4$ \\
BA6 & $12.4( \pm 1.7)$ & $>100$ & $>8$ \\
BA8 & $13.2( \pm 1.6)$ & $>100$ & $>7.6$ \\
Bdz & $13.5( \pm 1.3)$ & $>100$ & $>7.4$ \\
\hline
\end{tabular}

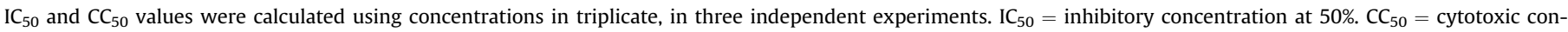
centration at $50 \%$. S.D. $=$ standard deviation. $\mathrm{Bdz}=$ benznidazole.

a Cells were exposed to derivatives for $6 \mathrm{~h}$ and activity was determined 3 days after incubation with derivatives.

b Cell viability of BALB/c mouse macrophages determined $6 \mathrm{~h}$ after treatment.

c $\mathrm{SI}$ is selectivity index, calculated by the ratio of $\mathrm{CC}_{50}$ (macrophages) and $\mathrm{IC}_{50}$ (amastigotes). 


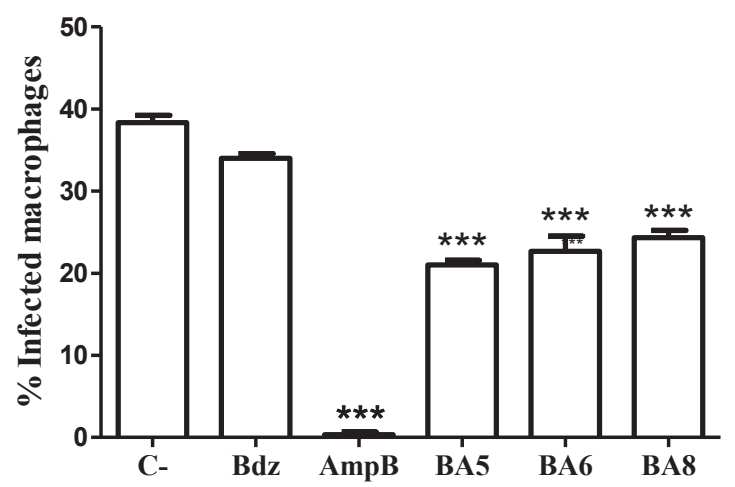

Fig. 5. The betulinic acid derivatives impair $T$. cruzi trypomastigote invasion in macrophages. Mouse macrophages were simultaneously infected with $10^{7} \mathrm{Y}$ strain trypomastigotes and treated with betulinic acid derivatives $(50 \mu \mathrm{M})$ or benznidazole $(50 \mu \mathrm{M})$ for $2 \mathrm{~h}$. $\mathrm{Bdz}=$ benznidazole; $\mathrm{AmpB}=$ amphotericin B. ${ }^{* * *}, P<0.001$ compared with untreated cultures.

betulinic acid derivatives was performed to investigate the piperidinyl moiety attached to the C28 position of carboxylic acid function, varying thus the hydrogen-bond sites. In fact, most of semisynthetic derivatives had $\mathrm{IC}_{50}$ values lower than betulinic acid and, in some cases (BA4, BA5, BA6 and BA8), the values of IC $_{50}$ was also lower than benznidazole, the reference drug. More specifically, the attachment of morpholyl, thiomorpholyl or a 4-methylphenylpiperidine group produced the most potent compounds.

Next, the cytotoxicity of the compounds to mouse macrophages was analyzed. As shown in Table 1, all semi-synthetic derivatives presented values of $\mathrm{CC}_{50}$ higher than betulinic acid. The derivatives BA5 and BA8 showed the best profiles of selectivity with values of SI of 17.3 and 8.8 respectively.

\subsection{Investigating the mechanism of action}

We used scanning electron microscopy (SEM) to study the morphology of trypomastigotes treated or not with the most potent derivative (BA5). Untreated trypomastigotes had the typical elongated shape of the parasite without visible alterations in the plasma membrane or in cell volume (Fig. 2A). On the other hand, trypomastigotes treated for $24 \mathrm{~h}$ with BA5 ( 2 or $4 \mu \mathrm{M}$ ) had flagella retraction (Fig. 2D), loss of plasma membrane integrity and body deformation (Fig. 2G).

Transmission electron microscopy (TEM) was also used to examine ultrastructural alterations. Thin sections of untreated trypomastigotes observed by TEM revealed normal appearance of organelles, intact plasma membrane and cytoplasm without alterations (Fig. 2B). However, treatment with BA5 (2 or $4 \mu \mathrm{M}$ ) caused plasma membrane alterations (Fig. 2C), the formation of numerous atypical vacuoles within the cytoplasm (Fig. 2E), dilatation of Golgi cisternae (Fig. 2F) and profiles of endoplasmatic reticulum involving organelles as nucleus accompanied by the formation of autophagosomes (Fig. 2H and I).

To understand the mechanism by which compound BA5 causes parasite death, a double staining with annexin $\mathrm{V}$ and propidium iodide (PI) was performed for flow cytometry analysis. In untreated cultures, most cells were negative for annexin V and PI staining, demonstrating cell viability. In contrast, a significant increase in the number of PI-positive parasites was observed in cultures treated with BA5 at 5 and $10 \mu \mathrm{M}$. Treatment with $5 \mu \mathrm{M}$ of BA5 for $72 \mathrm{~h}$ resulted in $15.8 \%$ and $39.4 \%$ of cells positively stained for $\mathrm{PI}$ and $\mathrm{PI}^{+}$ annexin $\mathrm{V}^{+}$, respectively (Fig. 3). These results suggest that the BA5 derivative induces a necrotic process in Trypanosoma cruzi trypomastigotes.

\subsection{In vitro infection studies}

We next investigated the ability of BA4, BA5, BA6 and BA8

Table 3

Concentration reductions and combination indexes for anti-T. cruzi activity by BA5 and benznidazole on trypomastigotes and amastigotes forms of $T$. cruzi.

\begin{tabular}{|c|c|c|c|c|c|c|}
\hline \multirow[t]{2}{*}{ Compounds } & \multicolumn{2}{|c|}{$\begin{array}{l}\mathrm{IC}_{50} \pm \text { S.D }(\mu \mathrm{M})^{\mathrm{a}} \\
\text { Trypomastigotes }\end{array}$} & \multicolumn{2}{|c|}{$\begin{array}{l}\mathrm{IC}_{50} \pm \mathrm{S} . \mathrm{D}(\mu \mathrm{M})^{\mathrm{a}} \\
\text { Amastigotes }\end{array}$} & \multicolumn{2}{|l|}{$\mathrm{CI}^{\mathrm{b}}$} \\
\hline & Drug alone & Combination & Drug alone & Combination & Trypo & Ama. \\
\hline BA5 & $2.6 \pm 0.2$ & $0.9 \pm 0.1$ & $11.2 \pm 0.9$ & $3.7 \pm 0.8$ & $0.45 \pm 0.1$ & $0.84 \pm 0.1$ \\
\hline $\mathrm{Bdz}$ & $10.6 \pm 0.9$ & $1.3 \pm 0.4$ & $13.6 \pm 0.1$ & $5.8 \pm 0.6$ & & \\
\hline
\end{tabular}

${ }^{\mathrm{a}} \mathrm{IC}_{50}$ values were calculated using concentrations in triplicates and two independent experiments were performed.

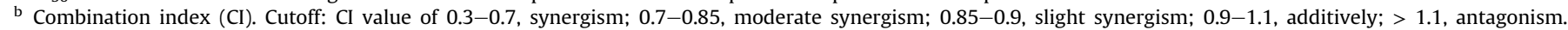
S.D = standard deviation.

A

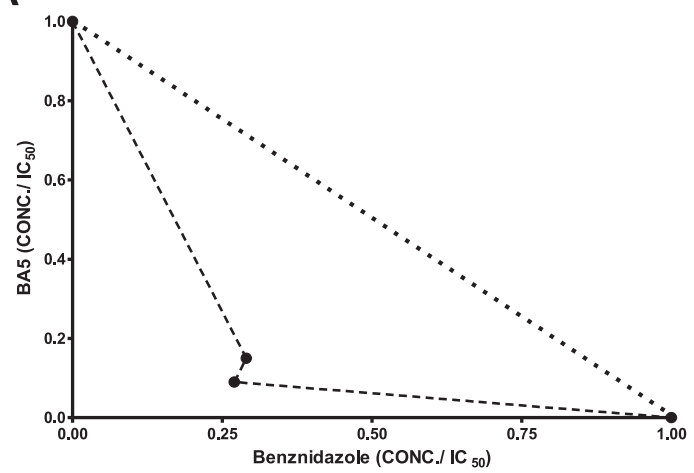

B

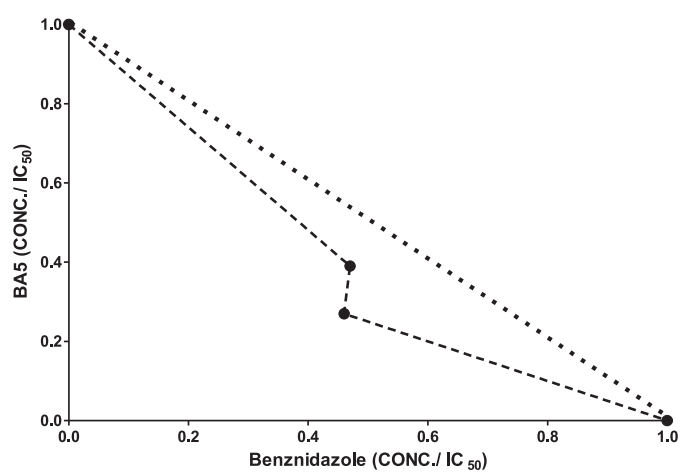

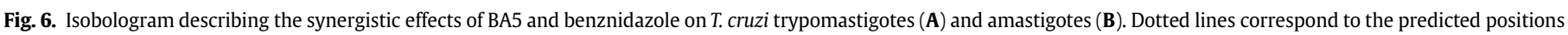
of the experimental points for additive effects. 
derivatives to inhibit the development of parasites in host cells. Macrophages infected with Trypanosoma cruzi trypomastigotes treated with $50 \mu \mathrm{M}$ of the compounds were stained with hematoxylin and eosin for analysis by optical microscopy. As shown in Fig. 4, treatment with the semi-synthetic compounds significantly decreased the percentage of infected macrophages $(P<0.001)$ and the relative number of amastigotes per 100 macrophages $(P<0.001)$ when compared with untreated cultures. When tested at different concentrations, it was possible to calculate the $\mathrm{IC}_{50}$ value of the derivatives against intracellular parasites. As shown in Table 2, the derivatives had an equipotent activity when compared to benznidazole. Cytotoxicity measured at $6 \mathrm{~h}$ of drug exposure demonstrated that benznidazole, as well as all derivatives testes are not cytotoxic at the tested concentrations $\left(\mathrm{CC}_{50}>100 \mu \mathrm{M}\right)$. These data demonstrate that the derivatives are selective compounds.

We also evaluated the effect of the most potent derivatives on the invasion process. In this assay, peritoneal macrophages were exposed to trypomastigotes and at the same time treated with the semi-synthetic BA5, BA6 and BA8 derivatives $(50 \mu \mathrm{M})$ for $2 \mathrm{~h}$. After this time, the cells were washed with saline solution to remove extracellular parasites and incubated for 2 additional hours. Cells were stained with hematoxylin and eosin and analyzed by optical microscopy. Amphotericin B was used as a positive control for this experiment. As shown in Fig. 5, the derivatives significantly inhibited the parasite invasion in comparison to untreated cells $(P<0.001)$, but not as efficiently as the positive control amphotericin $\mathrm{B}$, although they were more effective than benznidazole, that show no significant activity on this assay.

Finally, the antiparasitic effect of BA5 and benznidazole in combination was investigated against trypomastigote and amastigote the forms of Trypanosoma cruzi. In comparison to the drugs alone, the combination of BA5 and benznidazole reduced $\mathrm{IC}_{50}$ values in both forms of the parasite. In fact, the IC $\mathrm{C}_{50}$ of benznidazole decreased in average by $88 \%$ and $43 \%$ when combined with EEPA BA5 against trypomastigotes and amastigotes forms of $T$. cruzi respectively (Table 3 ).

An isobologram analysis and CI calculation, which can distinguish between the synergistic, additive and antagonistic effects of two compounds, confirmed that the combination of BA5 and benznidazole resulted in a remarkable synergistic effect on trypomastigotes and a moderate synergistic effect on amastigotes (Table 3; Fig. 6).

\section{Discussion}

Terpenoids, such as betulinic acid, are some of the most investigated source of antiparasitic compounds in terms of potency and selectivity (Newman and Cragg, 2012). In the present study, we investigated the anti-Trypanosoma cruzi activity of amide semisynthetic betulinic acid derivatives containing substituents attached in the lupane backbone. We observed that an incorporation of an amide on C-28 enhanced the anti-T. cruzi activity. This led to the identification of compound BA5, which exhibited a potency superior to benznidazole, the current standard drug. Several reports describe the chemical modifications of betulinic acid at C-28 position to produce semi-synthetic derivatives with enhanced antimalarial, anti-tumor and anti-viral activities (Jeong et al., 1999; Baltina et al., 2003; Domínguez-Carmona et al., 2010). This is the first report, however, regarding the contribution of the incorporation of amides on C-28 as drug design strategy to enhance the antiT. cruzi activity.

An examination of parasite morphology revealed that compound BA5 is parasiticidal, by altering parasite ultrastructure. It induced flagella retraction, loss of plasma membrane integrity and notable cell body deformation. Interestingly, the treatment with
BA5 also led to the formation of numerous and atypical vacuoles within the cytoplasm, as well as the dilatation of Golgi cisternae. Lack of membrane integrity and cytoplasmic vacuolization are often associated to necrotic parasitic death (Rodriguez et al., 2006; Zong and Thompson, 2006). Through flow cytometry assays, we confirmed the parasitic death by necrosis. In addition, we observed that the treatment produced endoplasmatic reticulum profiles involving organelles as nucleus accompanied by the formation of autophagosomes, typical features of autophagy (Tsujimoto and Shimizu, 2005; Fernandes et al., 2012).

Most importantly, the semi-synthetic derivatives were able to prevent the parasite development and invasion into host cells, crucial events for $T$. cruzi infection establishment, with potency similar to benznidazole. As a limitation, these compounds didn't eliminate intracellular amastigotes even in the highest concentration tested. However, the derivative BA5 exhibited synergistic activity when used in combination to benznidazole. These results encourage further investigations, since the combination of drugs is becoming increasingly attractive to combat parasitic diseases (Alirol et al., 2013; Diniz et al., 2013; Sousa et al., 2014).

Altogether, these findings reinforce that terpenoids are potent and selective trypanocidal agents. Therefore, the screening for structurally-related BA5 derivative for Chagas disease treatment is an attractive line of drug development.

\section{Conclusion}

Here, we have reported the synthesis and anti-T. cruzi evaluation of betulinic acid and its new amide semi-synthetic derivatives. By varying substituents attached to the amide group, we could find substituents that retain, enhance or greatly increase the trypanocidal activity, in comparison to betulinic acid. Specifically, we identified the betulinic acid derivative BA5 as a selective anti-T. cruzi agent, which destroys parasite cells by necrotic death and acts synergistically in combination to benznidazole.

\section{Acknowledgements}

This work was supported by grants from CNPq (grant number 562655/2010-7), PRONEX (grant number 0002/2014), FAPESB (grant number 0042/2013) and FINEP (grant number 01.04.0320$00)$.

\section{References}

Alakurtti, S., Heiska, T., Kiriaziz, A., Sacerdoti-Sierra, N., Jaffe, C.L., Yli-Kauhaluoma, J. 2010. Synthesis and anti-leishmanial activity of heterocyclic betulin derivatives Bioorg. Med. Chem. 18, 1573-1582.

Alirol, E., Schrumpf, D., Amici-Heradi, J., Riedel, A., de Patoul, C, Quere, M. Chappuis, F., 2013. Nifurtimox-eflornithine combination therapy for secondstage gambiense human African trypanosomiasis: Médecins Sans Frontiéres experience in the Democratic Republic of the Congo. Clin. Infect. Dis. 56, 195-203.

Baglin, I., Mitaine-Offer, A.C., Nour, M., Tan, K., Cave, C., Lacaile-Dubois, M.A., 2003 A review of natural and modified betulinic, ursolic and echinocystic acid derivatives as potential antitumor and anti-HIV agents. Mini Rev. Med. Chem. 3 525-539.

Baltina, L.A., Flekhter, O.B., Nigmatullina, L.R., Boreko, E.I., Pavlova, N.I. Nikolaeva, S.N., Savinova, O.V., Tolstikov, G.A., 2003. Lupane triterpenes and derivatives with antiviral activity. Bioorg. Med. Chem. Lett. 13, 3549-3552.

Barbosa-Filho, J.M., Trigueiro, J.A., Cheriyan, U.O., Bhattacharyya, J., 1985. Constituents of the stem-bark of Ziziphus joazeiro. J. Nat. Prod. 48, 152-152.

Chen, Y., Li, S., Sun, F., Han, H., Zhang, X., Fan, Y., Tai, G., Zhou, Y., 2010. In vivo antimalarial activities of glycoalkaloids isolated from Solanaceae plants. Pharm. Biol. 48, 1018-1024.

Chakraborty, B., Dutta, D., Mukherjee, S., Das, S., Maiti, C., Das, P., Chowdhury, C. 2015. Synthesis and biological evaluation of a novel betulinic acid derivative as a inducer of apoptosis in human colon carcinoma cells (HT-29). Eur. J. Med. Chem. 102, 93-105. 
Chandramu, C., Monohar, R.D., Krupadanam, D.G., Dashavantha, R.V., 2003. Isolation, characterization and biological activity of betulinic acid and ursolic acid from Vitex negundo I. Phytother. Res. 27, 129-134.

Chou, T.C., Talalay, P., 2005. Quantitative analysis of dose-effect relation-ships: the combined effects of multiple drugs or enzyme inhibitors. Adv. Enzyme Regul. 22, 27-55.

Costa, J.F.O., Barbosa-Filho, J.M., Maia, G.L.A., Guimarães, E.T., Meira, C.S., Ribeirodos-Santos, R., Carvalho, L.C.P., Soares, M.B.P., 2014. Potent anti-inflammatory activity of betulinic acid treatment in a model of lethal endotoxemia. Int. Imunopharmacol. 23, 469-474.

Da Silva, C.F., Junqueira, A., Lima, M.M., Romanha, A.J., Junior, P.A.S., Stephens, C.E. Som, P., Boykin, D.W., Soeiro, M.N.C., 2011. In vitro trypanocidal activity of DB745B and other novel arylimidamides against Trypanosoma cruzi. J. Antimicrobiol. Chemother. 66, 1295-1297.

Drag, M., Surowiak, P., Drag-Zelesinska, M., Dietel, M., Lage, H., Oleksyzyn, L., 2009 Comparison of the cytotoxicity effects of birch bark extract, betulin and betulinic acid towards human gastric carcinoma and pancreatic carcinoma drugsensitive and drug resistant cell lines. Molecules 14, 1639-1651.

Diniz, L.F., Urbina, J.A., De Andrade, I.M., Mazzeti, A.L., Martins, T.A., Caldas, I.S. Talvani, A., Ribeiro, I., Bahia, M.T., 2013. Benznidazole and posaconazole in experimental Chagas disease: positive interaction in concomitant and sequential treatments. PLoS Negl. Trop. Dis. 7, e2367.

Domínguez-Carmona, D.B., Escalante-Erosa, F., Gárcia-Sosa, K., Ruiz-Pinell, G., Gutierrez-Yapu, D., Chan-Bacab, M.J., Giménez-Turba, A., Peña-Rodríguez, L.M. 2010. Antiprotozoal activity of betulinic acid derivatives. Phytomedicine 17 , 379-382.

Fernandes, M.C., Da Silva, E.N., Pinto, A.V., De Castro, S.L., Menna-Barreto, R.F., 2012 A novel triazolic naphthofuranquinone induces autophagy in reservosomes and impairment of mitosis in Trypanosoma cruzi. Parasitology 139, 26-36.

Fivelman, Q.L. Adagu, I.S., Warhurst, D.G., 2004. Modified fixed-ratio isobologran method for studying in vitro interactions between atovaquone and proguanil or dihydroartemisinin against drug-resistant strain of Plasmodium falciparum. Antimicrob. Agents Chemother. 48, 4097-4102.

Gros, L., Lorente, S.O., Jimenez, C.J., Yardley, V., Rattray, L., Wharton, H., Little, S. Croft, S.L., Ruiz-Perez, L.M., Gonzalez-Pacanowska, D., Gilbert, I.H., 2006. Evaluation of azasterols as anti-parasitics. J. Med. Chem. 49, 6094-6103.

Hoet, S. Pieters, L., Muccioli, G.G., Habib-Jiwan, J.L., Opperdoes, F.R., QuetinLeclercq, J., 2007. Antitrypanosomal activity of triterpenoids and sterols from the leaves of Strychnos spinosa and related compounds. J. Nat. Prod. 70
1360-1363.

Innocente, A.M. Silva, G.N., Cruz, L.N., Moraes, M.S., Nakabashi, M., Sonnet, P. Gosmann, G., Garcia, C.R., Gnoatto, S.C., 2012. Synthesis and antiplasmodial activity of betulinic acid and ursolic acid analogues. Molecules 17, 12003-12014.

Jeong, H.J., Chai, H.B., Park, S.Y., Kim, D.S., 1999. Preparation of amino acid conjugates of betulinic acid with activity against human melanoma. Bioorg. Med. Chem. Lett. 9 (8), 1201-1204.

Morillo, C.A., Marin-Neto, J.A., Avezum, A., et al., 2015. Randomized trial of benznidazole for chronic chagas'cardiomyopathy. N. Engl. J. Med. 373, 1295-1306.

Newman, D.J., Cragg, G.M., 2012. Natural products as sources of new drugs over the 30 years from 1981 to 2010. J. Nat. Prod. 75, 311-335.

Pinto-Dias, J.C., 2006. The treatment of Chagas disease (South American trypanosomiasis). Ann. Intern. Med. 144, 722-774.

Rassi Jr., A., Rassi, A., Marin-Neto, J.A., 2010. Chagas disease. Lancet 375, 1388-1402.

Rodrigues, J.C., Seabra, S.H., De Souza, W., 2006. Apoptosis-like death in parasitic protozoa. Braz. J. Morphol. Sci. 23, 87-98.

Soares, M.B.P., Silva, C.V., Bastos, T.M., Guimarães, E.T., Figueira, C.P., Smirlis, D., Azevedo Jr., W.F., 2012. Anti-Trypanosoma cruzi activity of nicotinamide. Acta Trop. 122, 224-229.

Sousa, M.C., Varandas, R., Santos, R.C., Santos-Rosa, M., Alvez, V., 2014. Antileishmanial activity of semisynthetic lupane triterpenoids betulin and betulinic acid derivatives: synergic effects with miltefosine. PLoS One 9, e89939.

Spivak, A.Y., Keiser, J., Vargas, M., Gubaidullin, R.R., Nedopekina, D.A., Shakurova, E.R., Khalitova, R.R., Odinokov, V.N., 2014. Synthesis and activity of new triphenylphosphonium derivatives of betulin and betulinic acid against Schistosoma mansoni in vitro and in vivo. Bioorg. Med. Chem. 22, 6297-5304.

Tsujimoto, Y., Shimizu, S., 2005. Another way to die: autophagic programmed cell death. Cell Death Differ. 12, 1528-1534.

Urbina, J.A., Docampo, R., 2003. Specific chemotherapy of Chagas disease: controversies and advances. Trends Parasitol. 19, 495-501.

Viotti, R., Vigliano, C., Lococo, B., Alvarez, M.G., Petti, M., Bertocchi, G., Armenti, A., 2009. Side effects of benznidazole as treatment in chronic Chagas disease: fears and realities. Expert Rev. Anti Infect. Ther. 7, 157-163.

Yin, M.C., 2015. Inhibitory effects and actions of pentacyclic triterpenes upon glycation. Biomed. (Taipei) 5, 13.

Yogeeswiswari, P., Sriram, D., 2005. Betulinic acid and its derivatives: a review on their biological properties. Curr. Med. Chem. 12, 657-666.

Zong, W.X., Thompson, C.B., 2006. Necrotic death as a cell fate. Genes Dev. 20,1-15. 